\title{
A Comparison of Effective Discretization Schemes for The Double Heston Model in Financial Industry
}

\author{
Y.F. Sun, L.T. Ding, C.Y. Liu, G.Y. Zhang \\ School of Management, Guangdong University of Technology \\ Guangzhou, China
}

\begin{abstract}
This article applies four popular discretization schemes, i.e. Andersen's quadratic exponential (QE) scheme, Zhu's scheme, semi-analytical (SA) scheme, and Alfonsi's second-order scheme, to numerically simulate the double Heston stochastic volatility model. We compare the quality of these schemes in paths simulating by measuring the accuracy in option pricing, with reference values offered by the Fourier COS expansion method (namely the COS method, proposed by F. Fang \& C. W. Oosterlee,2008). Numerical results show that not all of these widely used schemes are of acceptable quality in simulating the asset paths when both the Feller conditions in the stochastic volatility model are not satisfied.
\end{abstract}

Keywords-heston stochastic volatility model; discretization scheme; option pricing; cos method; feller conditions

\section{INTRODUCTION}

An increasing amount of literature suggest that the double Heston stochastic volatility (hereinafter, $\mathrm{dbH}$ ) model is potentially a good financial model and expected to have an encouraging future in option pricing and risk hedging[1]. However, an exact simulation to the $\mathrm{dbH}$ model is proved to be impossible. The existing effective disretization schemes are the quadratic exponential (hereinafter, QE) scheme[2], Zhu's scheme[3] and Alfonsi's second-order scheme[4], and etc. All schemes mentioned above claim that they can achieve good accuracy in simulating the Heston stochastic volatility model(see[5]). The motivate of this research is to find a genuinely qualified disretization scheme for the $\mathrm{dbH}$ model under any censorious condition. To this end, we compare all schemes' performances in simulating the $\mathrm{dbH}$ model on the same ground (i.e., using the same parameters of model, the same time step and controlling variables in experiments on the same PC), from a common but trivial test to the most severe check.

\section{MODEL PRESENTATION}

As an ordinary extension of the Heston model, the $\mathrm{dbH}$ model(see [6]), under the risk-neutral ${ }^{Q}$-measure, is defined by two independent variance processes, i.e.

$$
\left\{\begin{array}{l}
d S(t) / S(t)=(r-q) d t+\sqrt{V^{1}(t)} d W_{1}^{S}(t)+\sqrt{V^{2}(t)} d W_{2}^{S}(t), \\
d V^{1}(t)=\kappa_{1}\left(\theta_{1}-V^{1}(t)\right) d t+\xi_{1} \sqrt{V^{1}(t)} d W^{V^{1}}(t), \\
d V^{2}(t)=\kappa_{2}\left(\theta_{2}-V^{2}(t)\right) d t+\xi_{2} \sqrt{V^{2}(t)} d W^{V^{2}}(t) .
\end{array}\right.
$$

where $r$ is the risk-free rate of return; $q$ is the yields flow rate; $\theta$ is the long variance, $\kappa$. is the rate at which $V^{\cdot}(t)$ reverts to $\theta$; $\xi$ is the volatility of the volatility which governs the variance of $V^{\cdot}(t) ; W^{S}(t), W^{V}(t)$ are Wiener processes, with correlation coefficient $\rho$.

\section{DISCRETIZATION SCHEMES FOR THE DBH MODEL}

For comprehensiveness of content, we summarize the famous QE scheme, Zhu's scheme, SA scheme, and Alfonsi's second-order scheme in this section. More details can be found in Andersen (2008),Zhu(2011), Alfonsi(2010), Sun, Zhang \& Li(2014) [7] and references therein.

\section{A. Discretization Scheme for the Asset Price Process}

For the price process, the predictor-corrector scheme for the log-price under the double Heston model can be written as (see [2][7]):

$$
\log \left(\hat{S}_{t+\Delta}\right)=\log \left(\hat{S}_{t}\right)+r \Delta+\sum_{j=1,2} K_{0}^{j}+K_{1}^{j} \hat{V}_{t}^{j}+K_{2}^{j} \hat{V}_{t+\Delta}^{j}+\sqrt{K_{3}^{j} \hat{V}_{t}^{j}+K_{4}^{j} \hat{V}_{t+\Delta}^{j} Y_{j}},
$$

where

$$
\begin{gathered}
K_{0}^{j}=-\frac{\rho_{j} \kappa_{j} \theta_{j}}{\xi_{j}} \Delta, K_{1}^{j}=\gamma_{1} \Delta\left(\frac{\kappa_{j} \rho_{j}}{\xi_{j}}-\frac{1}{2}\right)-\frac{\rho_{j}}{\xi_{j}}, K_{2}^{j}=\gamma_{2} \Delta\left(\frac{\kappa_{j} \rho_{j}}{\xi_{j}}-\frac{1}{2}\right)+\frac{\rho_{j}}{\xi_{j}}, K_{3}^{j}=\gamma_{1} \Delta(1 \\
\left.-\rho_{j}^{2}\right), K_{4}^{j}=\gamma_{2} \Delta\left(1-\rho_{j}^{2}\right) \text { and } \gamma_{1}+\gamma_{2}=1, \gamma_{1}, \gamma_{2} \in[0,1], Y_{j}, j=1,2 \text { are }
\end{gathered}
$$

independent standard Gaussian random variables, independent of those in the variance processes.

\section{B. Discretization Scheme for the Variance Process}

1) The QE scheme

$$
\hat{V}_{t+\Delta}^{j}=1_{\Psi_{j} \leq \Psi_{c}}\left[a_{j}\left(b_{j}+Z_{j}\right)^{2}\right]+1_{\Psi_{j}>\Psi_{c}}\left[1_{p_{j}<U_{j} \leq 1} \beta_{j}^{-1} \log \left(\frac{1-p_{j}}{1-U_{j}}\right)\right]
$$

where

$$
\begin{aligned}
& a_{j}=\frac{m_{j}}{1+b_{j}^{2}}, b_{j}=2 \Psi_{j}^{-1}-1+\sqrt{2 \Psi_{j}^{-1}\left(2 \Psi_{j}^{-1}-1\right)}, \Psi_{j}=\frac{s_{j}^{2}}{m_{j}^{2}}, \\
& m_{j}=\theta_{j}+\left(\hat{V}_{t}^{j}-\theta_{j}\right) e^{-\kappa_{j} \Delta}, \\
& s_{j}^{2}=\frac{\hat{V}_{t}^{j} \xi_{j}^{2} e^{-\kappa_{j} \Delta}}{\kappa_{j}}\left(1-e^{-\kappa_{j} \Delta}\right)+\frac{\theta_{j} \xi_{j}^{2}}{2 \kappa_{j}}\left(1-e^{-\kappa_{j} \Delta}\right)^{2}, p_{j}=\frac{\Psi_{j}-1}{\Psi_{j}+1}, \beta_{j}=\frac{1-p_{j}}{m_{j}}
\end{aligned}
$$

and $Z_{j}(j=1,2)$ is a

standard Gaussian random variable independent of each other; $U_{j}(j=1,2) \in[0,1]$ is an independent uniform random number.

2) The Zhu's scheme: Define new variables $v_{t}^{j}=\sqrt{V_{t}^{j}}$ (where $j=1,2)$. The Zhu's scheme for the variance process writes $\hat{v}_{t+\Delta}^{j}=\hat{v}_{t}^{j}+\frac{\kappa_{j}}{2}\left(\hat{\lambda}_{j}(t)-\hat{v}_{t}^{j}\right) \Delta+\frac{\xi_{j}}{2} \sqrt{\Delta} Z_{j}$ and $\hat{V}_{t+\Delta}^{j}=\left(\hat{V}_{j+\Delta}^{j}\right)^{2}$ $\hat{\lambda}_{k}^{j}=\frac{1}{1-e^{-\frac{\kappa_{j}}{2} \Delta}}\left[\sqrt{-\frac{\xi_{j}^{2}}{4 \kappa_{j}}\left(1-e^{-\kappa_{j} \Delta}\right)+\theta_{j}+\left(V_{t_{k}}^{j}-\theta_{j}\right) e^{-\kappa_{j} \Delta}}-\hat{v}_{t_{k}}^{j} e^{-\frac{\kappa_{j}}{2} \Delta}\right]$ and 
$Z_{j}(\quad j=1,2) \quad$ is a standard Gaussian random variable independent of each other.

3) The Semi-analytical scheme: In the exact simulation scheme (Broadie \& Kaya (2006)[8]), the variance process was well approximated by $\hat{V}_{t+\Delta}^{j} \cong \frac{e^{k_{j}}}{n(\Delta)} \chi_{d_{j}}^{\prime 2}\left(\lambda_{j}\right)$, where $n_{j}(\Delta)=\frac{4 \kappa_{j} e}{\varepsilon^{2}\left(1-\kappa_{i} \Delta\right.}, \lambda_{j}=n_{j}(\Delta) \hat{V}_{t}^{j}, d_{j}=4 \kappa_{j}^{n_{j}}(\Delta) / \xi_{j}^{2}$. Further, the non-cen $\xi^{2}\left(1-e_{\text {chil }}^{-\kappa_{j} \Delta}\right)_{\text {squared }}$ distribution $\chi_{d}^{\prime 2}(\lambda)$ can be approximated by $(Z+\sqrt{\lambda})^{2}+\chi_{d-1}^{2}$ when $d>1$ and by $\chi_{d+2 N}^{2}$ when $d>0$, with $Z \square N(0,1), \chi_{v}^{2}$ an ordinary chi-squared distribution with $v$ degrees of freedom and where $N$ is Poisson distributed with mean $\lambda / 2$.

We combine the above variance sampling procedure and the price sampling formula (2) to form the semi-analytic (SA) Monte Carlo scheme. The difference between the SA scheme and the exact simulation scheme lies in the way sampling the integral of the variance process within time step $\Delta$. In Broadie \& Kaya (2006), this integral is calculated by a Fourier inversion method. However, numerical results in the literature have shown that the exact approach is computationally expensive (Broadie and Kaya, 2006), and have worse speedaccuracy trade-off than simpler schemes (Lord et al. 2010)[5].

Rather than using Fourier methods, the SA scheme applies the following rule to approximate to the integral: $\int_{t}^{t+\Delta} v(t) d t \approx \Delta\left[\gamma_{1} v(t)+\gamma_{2} v(t+\Delta)\right]$ for certain constants $\gamma_{1}$ and $\gamma_{2}$. There are multiple ways for setting $\gamma_{1}$ and $\gamma_{2}$, the simplest being the Euler-like setting $\gamma_{1}=1, \gamma_{2}=0$. A central discretization, on the other hand, would set $\gamma_{1}=\gamma_{2}=1 / 2$. In this research, we take the last one.
4) The second order Alfonsi's scheme (Alf2)

$$
\begin{gathered}
\hat{V}_{t+\Delta}^{j}=1_{\hat{V}_{t} \geq K_{j}(\Delta)}\left[e^{-\frac{\kappa_{j}}{2} \Delta}\left(\sqrt{\left(\kappa_{j} \theta_{j}-\frac{\xi_{j}^{2}}{4}\right) \Psi_{j}+e^{-\frac{\kappa_{j}}{2} \Delta} \hat{V}_{t}^{j}}+\frac{\xi_{j}}{2} \sqrt{\Delta} Z_{j}\right)^{2}+\left(\kappa_{j} \theta_{j}-\frac{\xi_{j}^{2}}{4}\right) \Psi_{j}\right]+ \\
1_{\hat{V}_{t}<K_{j}(\Delta)}\left[1_{U_{j} \leq \pi\left(\Delta, \hat{V}_{t}\right)} \frac{m_{j}\left(\Delta, \hat{V}_{t}\right)}{2 \pi\left(\Delta, \hat{V}_{t}\right)}+1_{U_{j}>\pi\left(\Delta, \hat{V}_{t}\right)} \frac{m_{j}\left(\Delta, \hat{V}_{t}\right)}{2\left(1-\pi\left(\Delta, \hat{V}_{t}\right)\right)}\right]
\end{gathered}
$$

where

$$
\begin{gathered}
K_{j}(\Delta)=1_{\xi_{j}^{2}>4 \kappa_{j} \theta_{j} e^{\frac{\kappa_{j}}{2}}}\left[\left(\frac{\xi_{1}^{2}}{4}-\kappa_{j} \theta_{j}\right) \Psi_{j}+e^{\frac{\kappa_{j}}{2} \Delta}\left(\sqrt{\left.\frac{\xi_{1}^{2}}{4}-\kappa_{j} \theta_{j}\right) \Psi_{j}}+\frac{\xi_{j}}{2} \sqrt{3 \Delta}\right)^{2}\right], \Psi_{j}=\frac{1-e^{-\frac{\kappa_{j}}{2}} \Delta}{\kappa_{j}}, \\
\pi\left(\Delta, \hat{V}_{t}\right)=\frac{1}{2}-\frac{1}{2} \sqrt{1-\frac{m_{j}^{2}\left(\Delta, \hat{V}_{t}\right)}{\operatorname{Var}_{j}\left(\Delta, \hat{V}_{t}\right)}}, m_{j}\left(\Delta, V_{t}\right)=E\left(V_{t+\Delta} \mid V_{t}\right), \operatorname{Var}_{j}\left(\Delta, V_{t}\right)
\end{gathered}
$$$$
=E\left(V_{t+\Delta}^{2} \mid V_{t}\right) \text {, and } Z_{j} \text { is a discrete random variable such that }
$$$$
P\left(Z_{j}=0\right)=\frac{2}{3}, P\left(Z_{j}=\sqrt{3}\right)=\frac{1}{6}, P\left(Z_{j}=-\sqrt{3}\right)=\frac{1}{6} \text {. }
$$

\section{NUMERCIAL EXPERIMENTS}

\section{A. Parameters Settings}

The difficulty of simulating the Heston-type model lies in the effective discretization to the variance process. Feller (1951) has proved that the variance process in the Heston model is strictly non-negative. Therefore, for rigorous check, we consider three increasingly difficult cases: 1) Case1: $\kappa_{1} \geq \frac{\xi_{1}^{2}}{2 \theta_{1}}, \kappa_{2} \geq \frac{\xi_{2}^{2}}{2 \theta_{2}} \quad$; 2) Case2: $\kappa_{1} \geq \frac{\xi_{1}^{2}}{2 \theta_{1}}, \kappa_{2}<\frac{\xi_{2}^{2}}{4 \theta_{2}}$; 3) Case3: $\kappa_{1}<\frac{\xi_{1}^{2}}{4 \theta_{1}}, \kappa_{2}<\frac{\xi_{2}^{2}}{4 \theta_{2}}$.

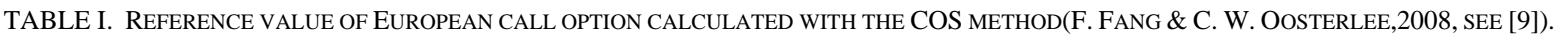

\begin{tabular}{ccccc}
\hline \multirow{2}{*}{ Strikes } & \multirow{2}{*}{ Term } & \multicolumn{3}{c}{ Reference Price } \\
\cline { 3 - 4 } & $T=1$ & Case1 & Case2 & 50.5082 \\
$100 \%$ & $T=1$ & 47.0316 & 39.0729 & 38.2680 \\
$130 \%$ & $T=1$ & 33.6541 & 36.3669 & 29.0825 \\
$70 \%$ & $T=30$ & 23.8377 & 26.9122 & 85.1216 \\
$100 \%$ & $T=30$ & 82.1304 & 83.2689 & 79.9137 \\
$130 \%$ & $T=30$ & 76.0970 & 77.5427 & 75.0317 \\
\hline
\end{tabular}

Case1 is equivalent to that both Feller conditions are satisfied, which makes Case 1 the easiest test among three cases. As one of Feller conditions is no longer satisfied, this makes discretization more challenging. The most difficult situation is Case3 where both Feller conditions are far from satisfied, which implies the most probability masses of $V_{t}^{1}$ and $V_{t}^{2}$ concentrate on the near of zero. Case 3 is then a rigorous test for all discretization schemes.

In experiments, we take: 1) $\kappa_{1}=0.9, \kappa_{2}=1.2 ; 2$ ) $\kappa_{1}=0.9, \kappa_{2}=0.3$; 3) $\kappa_{1}=0.2, \quad \kappa_{2}=0.3$ for above three Cases, respectively. The rest parameters in the $\mathrm{dbH}$ model are: $\theta_{1}=0.1, \theta_{2}=0.15, \quad V_{0}^{1}=0.6, V_{0}^{2}=0.7, \quad \xi_{1}=0.4, \xi_{2}=0.5, \quad \rho_{1}=-0.9$, $\rho_{2}=-0.9$. Besides, let $S_{0}=100 ; K=0.7 S 0, S 0,1.3 S_{0} ; r=0.03$, $T=1,30$ years. For QE scheme, $\gamma_{1}=0.5, \gamma_{2}=0.5$ and $\Psi_{c}=1.5$.

\section{B. Numerical Pricing Results}

Take Case 3 with $\Delta=1 / 8$ as an example. We plot the absolute bias curve (see Figure 1) for four discretization schemes, i.e., the QE scheme, Zhu's scheme, SA scheme and Alf2 scheme. Here, the bias bias is defined as the pricing differences between a discretization scheme and the COS method. To show how well these schemes works, we also provide the root-mean-squared (RMS) error curve in Figure 2 with $R M S=\sqrt{\text { bias }^{2}+\sigma_{f}^{2}}$ (wher $\sigma_{f}$ denotes as the standard error of payoff samples $\left.f\left(\hat{S}_{T}^{i}\right), i=1,2, \cdots, N_{\text {paths }}\right)$. Figure 2 shows that except the Zhu's scheme, the rest schemes have good convergences in option pricing in this example. 


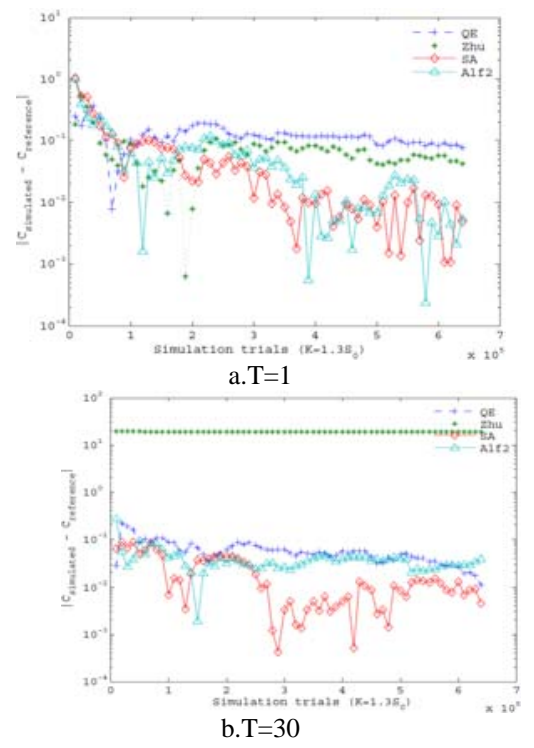

FIGURE I. ACCURACY OF PRICING SCHEMES FOR EUROPEAN CALL OPTION

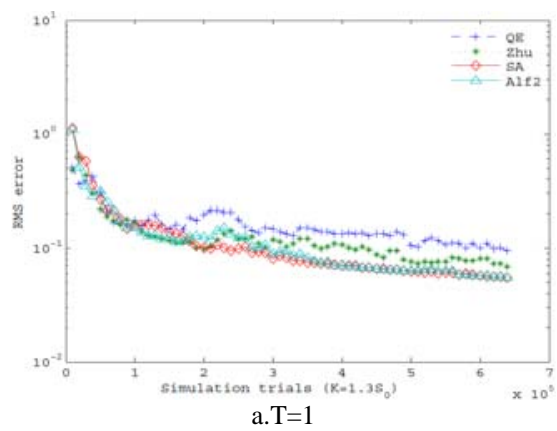

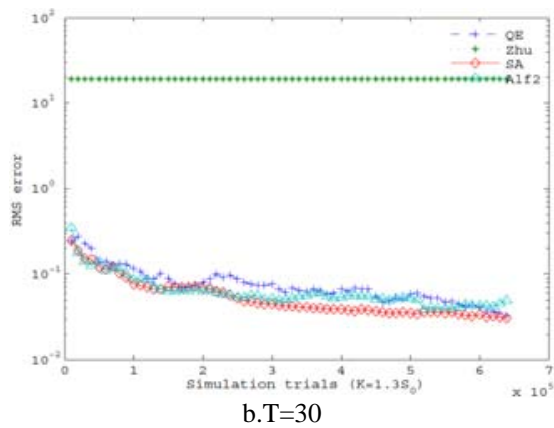

FIGURE II. CONVERGENCE OF DISCRETIZATION SCHEMES FOR EUROPEAN CALL OPTION.

Figure 1 shows that when the time step takes $\Delta=1 / 8$, the SA scheme and the Alfonsi's second-order scheme, are generally better than the QE and Zhu's scheme, when both Feller conditions in the dbH model are not satisfied. For the QE scheme, it would need a smaller time step $\Delta$ to achieve the same degree of accuracy as the SA scheme; while for Zhu's scheme, it fails to price rightly the option when the maturity is 30 -year (which also can be seen from Table 2).

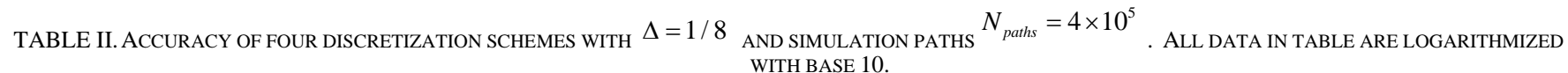

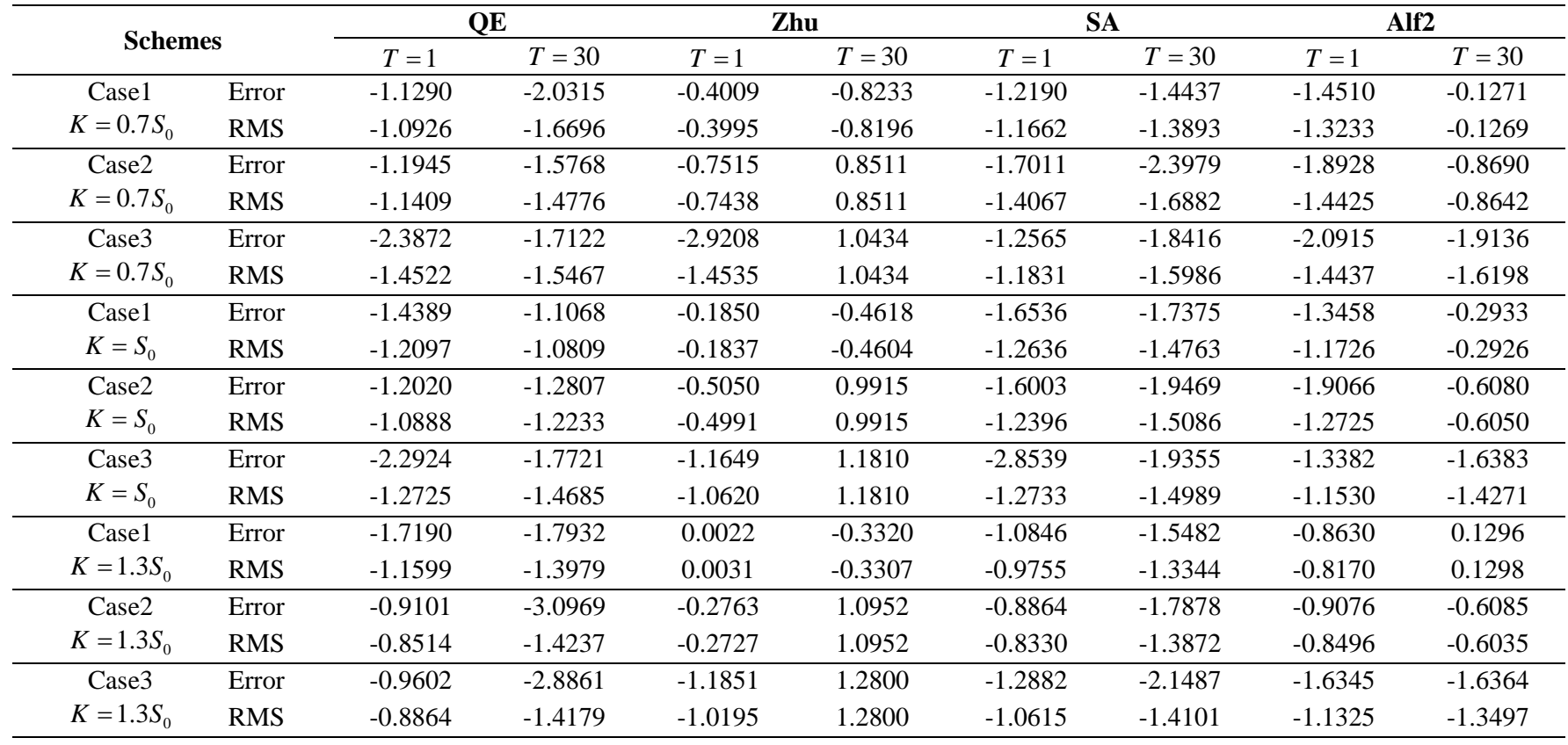


We also compare the efficiency of four computational schemes. Take Case 1 with $T=1$ for example. The CPU times for the QE, Zhu's, SA and Alf2 scheme are: 0.316, 0.2338, 1.4837 and 0.4757 in seconds, respectively. The implementation code is programmed with Matlab language and executed on a PC equipped with Win7(64bit) and Intel(R) Xeon(R) CPU E5-1620 v2 @3.70GHz 3.70GHz RAM 8.00GB. Though the efficiency of SA scheme is the lowest among all schemes, this cost is still acceptable if compared with that of the exact simulation method proposed by Broadie \& Kaya (2006) in which the cost is with hours in unit on the same PC.

\section{CONCLUSIONS AND FINAL REMARKS}

This article gives a simple but rigorous comparison for Andersen's QE scheme, Zhu's scheme, semi-analytical scheme, and Alfonsi's second-order scheme under the double Heston stochastic volatility model. Numerical results show that, the SA scheme is at least equally good as the QE scheme and Alfonsi's second-order scheme under any conditions. When the Feller conditions in the double Heston stochastic volatility model are not satisfied, the QE scheme may suffer from low accuracy if the time step is not small enough, while the Zhu's fails to correctly sample the asset path.

\section{ACKNOWLEDGEMENTS}

This work was supported by the NFS of Guangdong Province with grant titled "The Key Technologies of NearlyExact Simulation to Multi-Asset Heston Model: Distribution function and Implicit Solution Based Approaches”(2014).

\section{REFERENCES}

[1] Pricing Partners. Pricing Partners implements Double-Heston Model for its Equity Module, News and Events, 2009, 9. http://ww.pricingpartners.com/ news\&events/press-release/167.html

[2] Andersen, L.Simple and Efficient Simulation of the Heston Stochastic Volatility Model.Journal of Computational Finance, 11 (), pp.1-42, 2008.

[3] Jianwei Zhu: A Simple and Accurate Simulation Approach to the Heston Model, The Journal of Derivatives, 18(4), pp.26-36, 2011.

[4] Aurelien Alfonsi: High order discretization schemes for the CIR process: Application to affine term structure and Heston models, Math. Comp. 79, pp.209-237,2010.

[5] Lord, R., Koekkoek, R. and Van Dijk, D., A comparison of biased simulation schemes for stochastic volatility models, Quantitative Finance, 10(2), pp. 177-194, 2010.

[6] G., Pierre and P., Dylan, Efficient Simulation of the Double Heston model, SSRN Working Paper, July 18, 2009. http://papers.ssrn.com/sol3/papers. cfm?abstract_id=1434853

[7] Y.F. Sun, G.Y. Zhang and S.Q. Li. An Effective Simulation of Heston Model: Combining Quadratic Exponential and Exact Simulation schemes, WIT Transactions on Modelling and Simulation, 2014, forthcoming.

[8] Broadie, M., and Kaya, Ö.Exact Simulation of Stochastic Volatility and Other Affine Jump-Diffusion Processes. Operations Research,54(2), pp.217 -231, 2006.

[9] F.Fang and Cornelis W. Oosterlee. A novel pricing method for European option based on fourier-cosine series expansions. SIAM Journal of Scientific Computing, 31(2), pp.826-848, 2008. 